\title{
Retroelement-Linked H3K4me1 Histone Tags Uncover Regulatory Evolution Trends of Gene Enhancers and Feature Quickly Evolving Molecular Processes in Human Physiology
}

\author{
Daniil Nikitin ${ }^{1,2}$, Nikita Kolosov ${ }^{3}{ }^{(0}$, Anastasiia Murzina ${ }^{3}$, Karina Pats ${ }^{3}{ }^{(0}$, Anton Zamyatin ${ }^{3}\left({ }^{(}\right.$, \\ Victor Tkachev ${ }^{2}$, Maxim Sorokin 2,4, Philippe Kopylov ${ }^{4}$ and Anton Buzdin 1,2,4,* \\ 1 Group for Genomic Analysis of Cell Signaling Systems, Shemyakin-Ovchinnikov Institute of Bioorganic \\ Chemistry, 117997 Moscow, Russia; danya.nikitin.orel@gmail.com \\ 2 Omicsway Corp., Walnut, CA 91789, USA; tkachev@oncobox.com (V.T.); sorokin@oncobox.com (M.S.) \\ 3 ITMO University, 195251 Saint-Petersburg, Russia; nikita-kolosov@yandex.ru (N.K.); \\ murzinanastasiia@gmail.com (A.M.); karina.m.pats@gmail.com (K.P.); zamyatin.anton@gmail.com (A.Z.) \\ 4 Institute of Personalized Medicine, I.M. Sechenov First Moscow State Medical University, 119991 Moscow, \\ Russia; fjk@inbox.ru \\ * Correspondence: buzdin@oncobox.com
}

Received: 23 August 2019; Accepted: 1 October 2019; Published: 8 October 2019

\begin{abstract}
Background: Retroelements (REs) are mobile genetic elements comprising $40 \%$ of human DNA. They can reshape expression patterns of nearby genes by providing various regulatory sequences. The proportion of regulatory sequences held by REs can serve a measure of regulatory evolution rate of the respective genes and molecular pathways. Methods: We calculated RE-linked enrichment scores for individual genes and molecular pathways based on ENCODE project epigenome data for enhancer-specific histone modification H3K4me1 in five human cell lines. We identified consensus groups of molecular processes that are enriched and deficient in RE-linked H3K4me1 regulation. Results: We calculated H3K4me1 RE-linked enrichment scores for 24,070 human genes and 3095 molecular pathways. We ranked genes and pathways and identified those statistically significantly enriched and deficient in H3K4me1 RE-linked regulation. Conclusion: Non-coding RNA genes were statistically significantly enriched by RE-linked H3K4me1 regulatory modules, thus suggesting their high regulatory evolution rate. The processes of gene silencing by small RNAs, DNA metabolism/chromatin structure, sensory perception/neurotransmission and lipids metabolism showed signs of the fastest regulatory evolution, while the slowest processes were connected with immunity, protein ubiquitination/degradation, cell adhesion, migration and interaction, metals metabolism/ion transport, cell death, intracellular signaling pathways.
\end{abstract}

Keywords: human genome evolution; histone modifications; H3K4me1; enhancers of transcription; retrotransposons; retroelements; molecular pathways; gene ontology; epigenetics; gene expression regulation

\section{Introduction}

Transposable elements occupy nearly one-half of human genome [1]. Among them, retroelements (REs) form the most numerous and active class that shaped $~ 40 \%$ of human DNA [2]. REs impact human gene expression by providing functional regulatory regions including enhancers [3,4]. RE-linked enhancers act via recruiting of transcription factor proteins to the enclosed transcription factor binding sites (TFBS) [5,6]. Such enhancer activity of human REs has greatly influenced complex molecular processes such as innate immunity [7] and placentation [8], and RE-linked enhancers are currently recognized as one of the major driving forces of human regulatory evolution [9-11]. 
Deep sequencing-based technologies such as chromatin immunoprecipitation and sequencing (ChIP-seq) enabled whole genome studies of functional enhancer elements [12]. Monomethylation of H3 lysine 4 (H3K4me1) is major epigenetic mark of both active and dormant enhancers [13]. The whole genome profiles of H3K4me1 mark were resolved [14] and are widely used in gene regulation studies [15]. Investigating epigenomic data in an evolutionary context reveals a complex and constantly changing enhancer landscape [16]. RE-driven enhancers could affect animal diversity [17] and give birth to new genes [18] or even new tissues such as mammalian neocortex [19].

Recent studies of RE regulatory impact on the functioning of human molecular pathways [20,21] provide a computational framework to estimate regulatory evolution of major molecular processes by using RE-linked enhancer activity as the marker. The original analytic pipeline called RetroSpect [22] is based on mapping of RE-linked regulatory sites such as transcription factor binding sites (TFBS) in a close neighborhood of gene transcription start sites (TSSs) [20]. TSS-proximal regions are enriched in major cis-acting regulatory elements, and $10-\mathrm{Kb}$ interval proposed for the RetroSpect analysis covers a significant portion of RE-linked enhancers of human genes [23,24]. Comparing the ChIP-seq signal of RE-linked regulatory elements and of all regulatory elements in the 10-kb frame allows for the calculation of absolute and relative gene enrichments by RE-linked regulatory elements, or GRE and NGRE scores, respectively [21].

RetroSpect methodology enables calculation of relative and absolute RE-linked regulatory enrichment scores for both individual genes and intracellular molecular pathways, i.e. gene ensembles having a common molecular function [25]. After scoring individual genes according to the extent of their RE-linked regulation, the differential genes are then functionally annotated [26]. This annotation can be based on the calculation of molecular pathway activation scores as for gene expression studies [27], or on pathway instability metrics as for DNA mutation data [28]. In the RetroSpect pipeline, absolute and relative RE-linked regulatory enrichment scores for a given molecular pathway are calculated (PII and NPII scores, accordingly), which measure the strength of RE regulation in this pathway [21]. Data on topological structure of few thousands human molecular pathways are stored in public databases such as KEGG [29] and Reactome [30].

Alternatively, the gene set enrichment analysis can be applied which provides an independent way of biological annotation, e.g., based on Gene Ontology (GO) terms enrichment analysis as for the previous application of RetroSpect [31]. Comparison of the results obtained using pathway analysis and GO annotation enhances robustness of the RetroSpect analysis and allows for the reliable identification of consensus molecular processes that are enriched or deficient in RE-linked regulation. These processes, therefore, can be considered as those undergoing accelerated of delayed regulatory evolution [21].

Recent application of RetroSpect to RE-linked regulation of human molecular pathways at the level of TFBS for 13 human cell lines and 563 DNA-binding proteins showed that $\sim 55.5 \%$ of human TFBS were connected with REs [21]. Moreover, such molecular processes as gene regulation by microRNAs, olfaction, color vision, fertilization, cellular immune response, and amino acids and fatty acids metabolism and detoxication were found enriched by RE-linked TF regulation [21]. These observed patterns of RE-driven regulatory evolution delineate the most actively evolving-yet different-biological processes [9]. For example, RE-directed evolution of cellular immune response is connected with perpetual evolutionary arms-race between pathogens and hosts [32], whereas color vision is a recent evolutionary innovation of primates [33].

Further examination also evidenced that genes enriched by RE-linked TFBS showed a statistically higher proportion of miRNA and long non-coding RNA (lncRNA) genes than for the set of deficient genes [21].

In this study, we used RetroSpect to investigate RE-linked regulation of human genes by histone modification of H3K4me1, a well-known enhancer mark for the enclosing DNA. We extracted the whole genome of H3K4me1 ChIP-seq profiles for five human cell lines (MCF-7, K562, HepG2, HeLaS3, GM12878) from the ENCODE database. We found that only $27.8 \%$ of all H3K4me1 enhancer marks 
were associated with REs. This is twice lower compared to the previous figure of $55.5 \%$ of total RE-linked TFBS [21], which may reflect genome-wide epigenetic repression of RE-linked enhancers.

RetroSpect was then applied to detect their functional impacts. The major molecular processes enriched by RE-linked enhancer regulation were connected with DNA metabolism and maintenance of chromatin structure, sensory perception, neurotransmission and lipids metabolism. The least impacted processes, which may be considered the most conservatively regulated at the level of H3K4me1 [20], dealt with different aspects of the immune response, cell adhesion, migration and interaction, cell death, ion transport and various intracellular signaling pathways.

Moreover, among the top RE-linked regulation enriched genes we found statistically significantly higher proportion of genes coding miRNAs and lncRNAs. At the same time, the proportion of the lncRNA genes was significantly decreased in the bottom cohort of genes sorted by RE-linked H3K4me1 regulation.

\section{Materials and Methods}

\subsection{Identification of RE-Linked H3K4me1 Modification Tags}

Whole genome H3K4me1 ChIP-seq profiles for were extracted from the ENCODE database [34] for five human cell lines (K562, HepG2, GM12878, MCF-7, HeLa-S3) according to the standard ENCODE histone ChIP-seq protocol [35]. The reference human genome assembly 2009 (hg19) was indexed via Burrows-Wheeler algorithm using BWA software (version 0.7.10) [36]. Concatenation of fastq files with single-end or pairwise reads, alignment to the reference genome and filtering were done using BWA, Samtools (Sanger Institute, Hinxton, Cambridgeshire, UK, version 1.0), Picard (Broad Institute, Cambridge, MA, USA, version 1.92), Bedtools (Quinlan laboratory, University of Utah, UT, USA version 2.17.0), Phantompeakqualtools (Department of Genetics, Department of Computer Science, Stanford University, Stanford, CA, USA, version 1.1) and SPP (Department of Genetics, Department of Computer Science, Stanford University, Stanford, CA, USA, version 1.14) software [36]. Peak calling and signal generation were done using Macs (Harvard University, Cambridge, MA, USA, version 2.1.0) and Bedtools software [37] based on the alignment data. Aligned, filtered and normalized over control ChIP-seq reads for each cell line were mapped on the RE sequences annotated by RepeatMasker (Institute for Systems Biology, Seattle, WA, USA, version 3.2.7) [38] and downloaded from the UCSC Browser (RefGene table) [39]. The list of cell lines investigated here and raw ENCODE data files for each cell line is given in Supplementary Table S1.

\subsection{Gene Expression Data}

From the ENCODE database [34] we obtained RNA sequencing gene expression profiles for human cell lines using the following set of filters: "transcription", "total RNA-Seq" and "gene quantifications". For three out of five cell lines of interest, we found 19 experiments containing gene expression data in two technical replicates: 11 experiments for K562 cell line, 5 for HepG2 and 3 for GM12878. Accession numbers are shown in Supplementary Table S1.

\subsection{Measuring Gene Enrichment by RE-Linked H3K4me1 Histone Modification Tags}

The coordinates of human genes were downloaded from the USCS Browser (RefGenes table, genome assembly hg19) [39]. For each gene and cell line, all individual REs overlapping with the 10-kb neighborhood of its reference TSS were selected for further analysis. The 10-kb neighborhood covered an interval starting $5 \mathrm{~kb}$ upstream and ending $5 \mathrm{~kb}$ downstream the TSS. For every known gene in every cell line, we calculated total numbers of H3K4me1 histone modification tags mapped on either RE or RE-free loci in 10-kb neighborhood. We then calculated absolute (GRE) and relative (NGRE) RE-linked regulatory enrichment scores according to the formulas given in Supplementary Dataset 2 for 24,070 human genes. 


\subsection{Measuring Molecular Pathway Enrichment by RE-H3K4me1 Tags}

Gene architecture data of the molecular pathways were extracted from the databases BioCarta [40], KEGG [41], NCI [42], Reactome [43] and Pathway Central [44]. Pathways structure data were downloaded in .xml and. biopax formats from these databases and implemented in a computational algorithm Oncobox [21,27,45]. In every cell line, absolute (PII) and relative (NPII) RE-linked regulatory enrichment scores were calculated for 3095 molecular pathways according to the formulas given in Supplementary Dataset 2.

\subsection{Measuring Enrichment of Gene Sets by Non-Coding RNA Genes}

Significance of enrichment or deficiency of proportions of non-coding RNA genes in selected gene sets was evaluated using hyperbolic distribution separately for two classes of non-coding RNA: miRNA and lncRNA. For each gene set we used the following formula of hypergeometric probability mass function to calculate the probability values of numbers higher or lower than observed for miRNA or lncRNA classes:

$$
p(k, M, n, N)=\frac{\left(\begin{array}{l}
n \\
k
\end{array}\right)\left(\begin{array}{c}
M-n \\
N-k
\end{array}\right)}{\left(\begin{array}{c}
M \\
N
\end{array}\right)},
$$

where $M$ is the total number of genes, $N$ is the number of non-coding RNA genes in a gene set, $n$ is the number of genes in a gene set (e.g., top genes enriched or deficient in RE-linked regulation), $k$ is the number of non-coding RNA genes in a sample and $p(k, M, n, N)$ is the probability of observation of gene numbers $k, M, n, N$. Brackets denote binomial coefficients.

For each number of non-coding RNA genes $k$ we calculated probability $P(k, M, n, N)$ to observe a higher number of non-coding RNA according to the formula:

$$
P(k, M, n, N)=\sum_{i=k+1}^{n} p(i, M, n, N),
$$

where $p(i, M, n, N)$ is the hypergeometric probability mass function defined above.

We used $p<0.05$ as a significance threshold value for the hypothesis that non-coding RNA genes are overrepresented in a given gene set and $p>0.95$ for the hypothesis that non-coding RNA genes are not overrepresented.

Analogously, for each number of non-coding RNA genes $k$ we calculated probability $P^{\prime}(k, M, n, N)$ to observe a not higher number of non-coding RNA according to the formula:

$$
P^{\prime}(k, M, n, N)=\sum_{i=1}^{k} p(i, M, n, N),
$$

where $p(i, M, n, N)$ is the hypergeometric probability mass function defined above.

We used $p<0.05$ as a significance threshold value for the hypothesis that non-coding RNA genes are underrepresented in a given gene set and $p>0.95$ for the hypothesis that non-coding RNA genes are not underrepresented.

\subsection{Gene Ontology Enrichment Analysis}

Gene Ontology (GO) analysis of genes that are enriched or deficient in RE-linked H3K4me1 histone modification tags (RRE-enriched or RRE-deficient genes, respectively) was performed using DAVID (version 6.8) software [46] using human gene IDs extracted from USCS Genome Browser [47]. The p-values specifying the significance of observed GO-terms enrichment were calculated using a modified Fisher's exact test [48]. The cut-off for p-values significance was set as 0.05 . The enrichment 
values of GO-terms and Annotation Clusters were calculated as fold changes of their occurrences in the sample and in the human genome [48].

\subsection{Significance of Correlations}

The statistical significance of correlations was calculated as a Pearson correlation coefficient with a $p$-value using the python Seaborn (Michael Waskom, Center for Neural Science, NY, USA, version 0.9.0) package [49]. All calculations were carried out using Sklearn (French Institute for Research in Computer Science and Automation, Rocquencourt, France, version 0.21.2) module [50].

\subsection{Significance of Correlations}

To assess the confidence of the observed patterns for RE-impacted functional processes, we generated 500 sets of randomly permutated GRE and NGRE scores across the cell lines tested by randomly rearranging gene names. For each perturbation, we extracted a set of GRE-NGRE distribution-based 1204 top and bottom genes. These gene sets were profiled by DAVID (Laboratory of Human Retrovirology and Immunoinformatics, Applied/Developmental Research Directorate, Frederick National Laboratory for Cancer Research, MD, USA, version 6.8) software [46] and top-100 GO terms were selected for each set by the lowest $p$-value for each random permutation. Finally, we compared the distributions of $p$-values for the top-100 GO terms for the permuted and real gene sets: real RRE-enriched and RRE-deficient genes were respectively compared with the distributions of RRE-enriched and RRE-deficient genes in random permutations.

The overall RetroSpect data analysis pipeline is shown schematically in Supplementary File 3. All computational codes used here are freely available upon request to the authors.

\section{Results}

\subsection{Genes and Molecular Pathways Impacted by RE-Linked Histone Modification Marks}

In order to measure the impact of REs on gene regulation by H3K4me1 histone modifications we calculated GRE and NGRE scores for 24,070 human genes (Supplementary Table S4). These metrics were calculated separately for five cell lines MCF-7, K562, HepG2, HeLaS3 and GM12878 representing four different human tissues. Totally, 2,841,853 H3K4me1 histone modification tags were extracted from the ENCODE project repository including 482,894, 1,075,569, 705,632, 339,874 and 237,884, respectively, for the above human cell lines.

We then correlated these H3K4me1 histone modification profiles with the gene expression data obtained for the same cell lines (Figure 1). In this study, we considered RNA sequencing profiles because they are thought to represent the gold standard data in high throughput transcriptomic research [25]. In the ENCODE project repository, six profiles were available for cell line GM12878, 10 for cell line HepG2 and 22 for cell line K562. For the MCF-7 and HeLa-s3 cell lines, no RNA sequencing data were available. We observed a trend that H3K4me1 histone modification profiles positively correlated with the gene expression data (Figure 1). The highest correlations were observed for the long non-coding RNA genes (Figure 1D), the lowest was observed for microRNA genes (Figure 1C) and the intermediate pattern was observed for protein coding genes (Figure 1B). The highest correlations were detected between gene expression and histone modification profiles of the same cell lines (Figure 1). This confirmed that the H3K4me1 histone modification tags from the ENCODE database were related to their expected molecular functions.

We then tested whether gene-wise scores of RE-linked regulatory enrichments (GRE and NGRE) are comparable among the cell lines. We calculated pairwise gene-by-gene Pearson correlation coefficients between five cell lines investigated here for GRE (Figure 2A) or NGRE (Figure 2B) scores. 


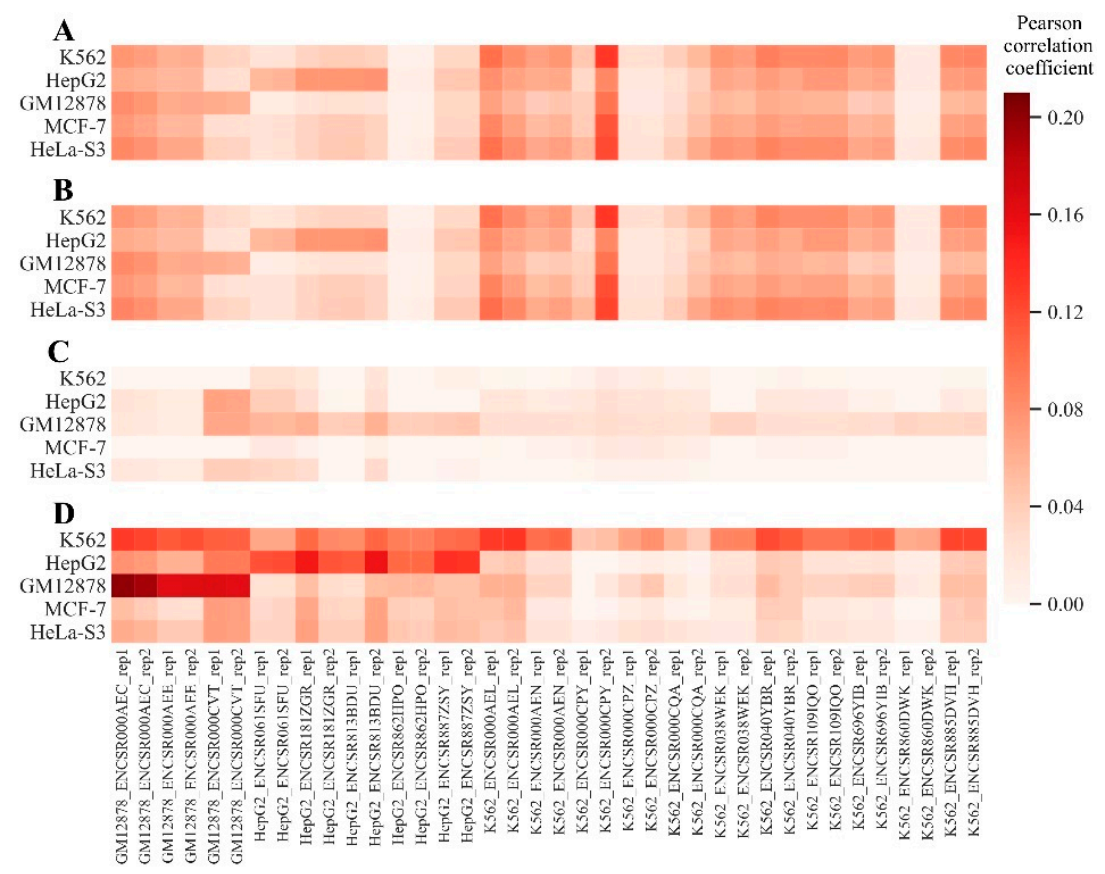

Figure 1. Correlations of $\mathrm{H} 3 \mathrm{~K} 4 \mathrm{me} 1$ histone modification profiles with the RNA sequencing gene expression data. The histone modification profiles (vertical axis) were compared with normalized gene expressions (abscissa) for 6 GM12878, 10 HepG2 and 22 K562 cell line RNA sequencing experiments. Pearson correlations were measured for (A) set of all known genes; (B) set of protein coding genes; (C) set of microRNA genes and (D) set of long non-coding RNA genes.

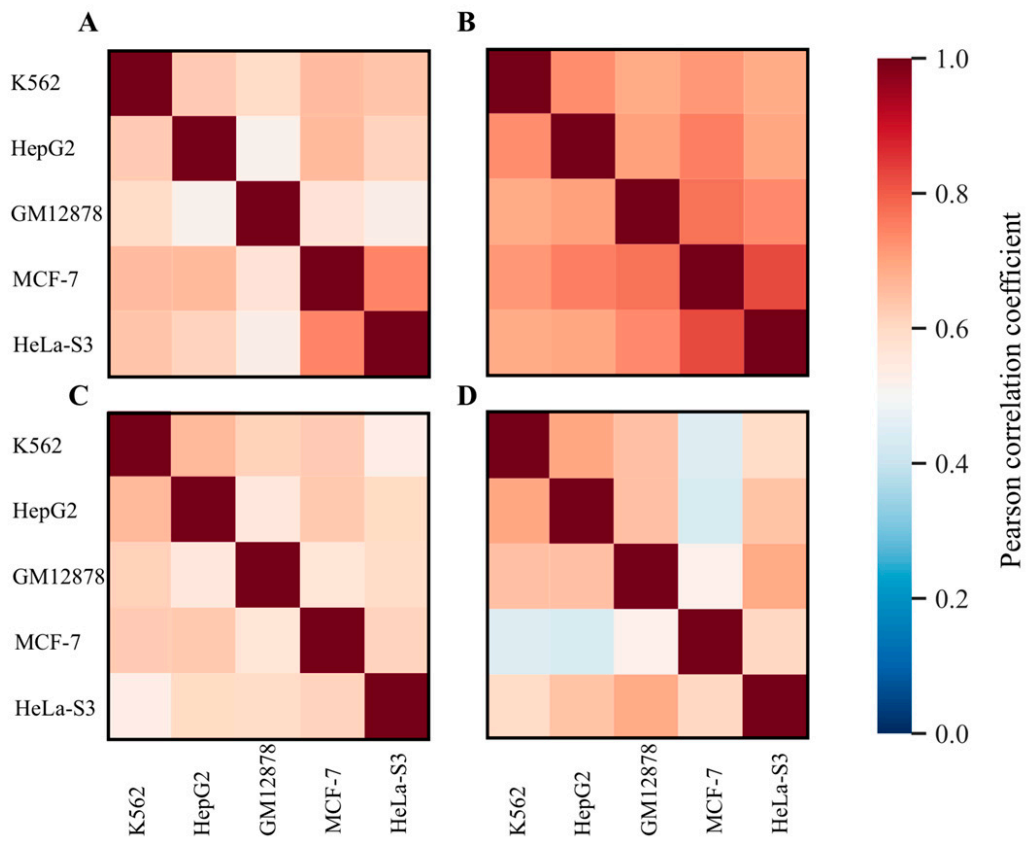

Figure 2. Comparison of RE-linked regulatory enrichment scores between five cell lines investigated. Each panel represents Pearson correlation plot for corresponding score, labels denote cell lines investigated. (A) GRE score; (B) NGRE score; (C) PII score and (D) NPII score. Color scale represents Pearson correlation. 
Gene-based scores showed a high degree of similarity between the cell lines: pairwise Pearson correlation coefficients were between 0.53 and 0.74 for GRE and between 0.68 and 0.83 for NGRE. Similarly, the PII and NPII scores also showed high similarities between the five different cell lines investigated (Figure 2C,D). We therefore concluded that gene-based scores were congruent among the cell lines tested and, for the next steps of the RetroSpect analysis, they were arithmetically averaged gene-by-gene across the cell lines.

We applied simple linear regression to identify genes that were relatively enriched of deficient by RE-linked H3K4me1 tags. To identify RE-linked regulation (RRE) enriched and deficient genes, we built a trend line using the least-square method in the coordinates of gene GRE and NGRE scores. Intercept of linear model was set to zero (Figure 3). The genes having greater relative (NGRE) compared to absolute (GRE) RE-regulation metric were considered RRE-enriched, and vice versa.

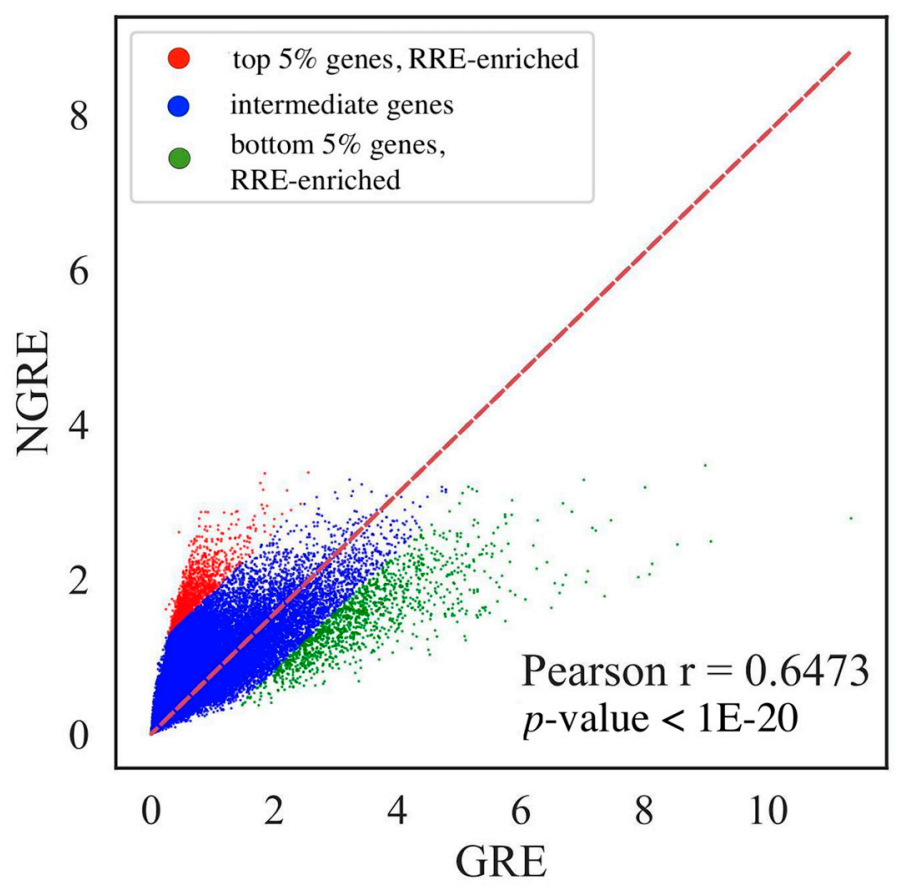

Figure 3. Comparison of GRE (abscissa axis) and NGRE (axis of ordinates) scores for human genes. Color indicates different gene groups (red-RRE-enriched genes, blue-intermediate genes, green-RRE-deficient genes). Each dot represents single gene. Pearson correlation coefficient (r) and Pearson $p$-value $(p)$ are shown separately.

For the above linear model, we sampled $5 \%$ of genes having biggest distances from the NGRE-GRE trend line. The above and below 5\% gene sets were considered, respectively, RRE-enriched and RRE-deficient (listed in Supplementary Table S5).

The same approach was also used for the molecular pathway data (Figures 2C,D and 4). We calculated absolute (PII) and normalized (NPII) pathway involvement scores for 3095 molecular pathways separately for five human cell lines (Supplementary Table S6). We then deduced pairwise Pearson correlation coefficients between the cell lines (Figure 2C,D respectively, for PII and NPII). Correlation coefficients for PII varied from 0.53 till 0.66, for NPII they varied from 0.44 till 0.69. Therefore, PII and NPII values were highly congruent between the cell lines investigated and for further analysis we used their averaged values across the five cell lines.

Similar to the gene-based analysis, we built a linear model and sampled the top and bottom 5\% molecular pathways with the biggest distances from the trend line and that were also considered RRE-enriched and RRE-deficient molecular pathways, accordingly (listed in Supplementary Table S7). 


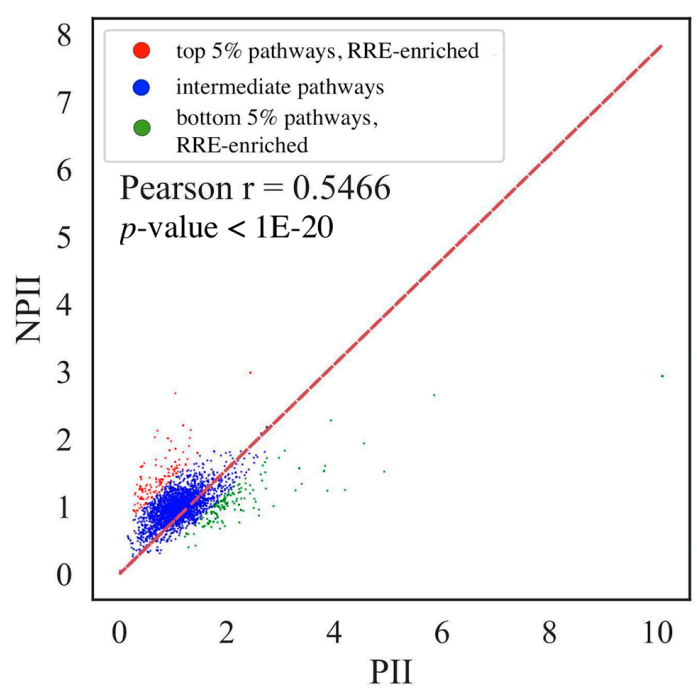

Figure 4. Comparison of PII scores (abscissa axis) and NPII scores (axis of ordinates) for human molecular pathways. Color indicates different pathway groups (red-RRE-enriched molecular pathways, blue-intermediate pathways, green-RRE-deficient molecular pathways). Each dot represents single pathway. Pearson correlation coefficient (r) and Pearson $p$-value $(p)$ are shown separately.

\subsection{Functional Characteristics of Top RRE-Enriched and Deficient Genes}

In order to functionally characterize RRE-enriched and deficient genes, we performed gene ontology analysis of the corresponding gene sets using DAVID software. We extracted all GO terms corresponding to molecular function, biological process and cellular component [51] and manually sorted them according to their biological roles.

\subsubsection{RRE-Enriched Genes}

For the RRE-enriched set of human genes (Supplementary Table S5) we defined nine major groups of the corresponding GO terms obtained using DAVID software (Supplementary Table S8). In toal, there were $32 \mathrm{GO}$ terms with a $p$-value less than 0.05 . Among them, six terms were connected with RNA synthesis and degradation, four were connected with DNA- and chromatin-linked processes, three were connected with translation, three were connected with metabolism of lipids, three were connected with metals metabolism and ion transport, two were connected with immune system, two were connected with sensory perception and neurotransmission, and two were connected with the cell cycle regulation. Finally, the last group called "Other and General terms" contained seven terms which solely represented particular biological functions.

\subsubsection{RRE-Deficient Genes}

For the RRE-deficient genes (Supplementary Table S5), we identified 16 groups of GO terms that passed the statistical threshold (Supplementary Table S8). This reflected a bigger number of p-value-filtered GO terms identified (169). Among them, 45 terms were connected with morphogenesis, 23 were connected with RNA synthesis and degradation, 15 were connected with different types of molecular signaling, four were connected with hormone signaling pathways, 11 were connected with programmed cell death, eight were connected with immunity, two were connected with protein ubiquitination and degradation, seven were connected with protein aggregation and import, 12 were connected with cell adhesion, migration and interaction, six were connected with cell cycle and mitosis regulation, four were connected with transport of ions, three were connected with DNA- and chromatin-linked processes, two were connected with response to stress, and two were connected with intracellular processes of response to viruses. Finally, the "Other and general terms" group contained eighteen different terms (Table 2). 


\subsubsection{Alternative GO Annotation Analysis}

Alternatively, we also did functional annotation of RRE-enriched (Supplementary File S9) and deficient (Supplementary File S10) gene sets using Gorilla software and "Gene Ontology Biological Process" database [52]. The only major biological process identified by Gorilla software analysis for the RRE-enriched gene set was gene silencing by microRNA and RNA catabolism; in turn, for the RRE-deficient gene set, (i) protein ubiquitination and (ii) negative regulation of TORC1 signaling processes were identified.

\subsubsection{Enrichment by Non-Coding RNA Genes}

MicroRNAs and lncRNAs are regulatory non-coding RNA molecules that can specifically modulate gene expression. We evaluated the contents of microRNA and IncRNA genes among the RRE-enriched and deficient genes. We used hypergeometric sampling model to calculate the degree of improbability to obtain the observed numbers of miRNA and lncRNA genes in the top and bottom gene sets by random sampling, summarized on Table 1.

Our findings suggested that both microRNA and lncRNA genes were strongly overrepresented in the group of RRE-enriched genes ( $p$-values 2.22e-08 and 3.49e-08, respectively). Moreover, lncRNA genes were also underrepresented in the RRE-deficient cohort ( $p$-value 0.0049), which was not the case for the microRNA genes ( $p$-value 0.33).

\subsubsection{Significance of RRE-Based Functional Gene Annotations}

In order to evaluate the significance of the observed annotations obtained using RRE characteristics, we generated 500 random permutations of gene names and corresponding GRE or NGRE scores. For each permutation, 1204 top and 1204 bottom genes were randomly taken and used as described above for the real RRE-top and bottom genes. These randomly generated gene sets were then analyzed using DAVID software, and top-100 GO terms were selected by the lowest $p$-value for each random permutation. Finally, we compared the distributions of $p$-values for the real and random gene sets (Figure 5A,B for RRE-enriched and deficient genes, respectively). This type of analysis showed that the RRE-deficient molecular processes identified here were mostly statistically significant, because the peak value of real distribution (mode) was lower than the mode of random distribution (Figure 5B). However, most of all RRE-enriched GO terms overlapped with the random distribution (Figure 5A), although there were few GO terms with significantly lower p-values than for the random permutations. Since none of the 500 random permutations generated GO-terms with $p$-values lower than those observed for the real RRE-enriched or RRE-deficient genes, the overall q-values of confidence for both groups were smaller than 0.002 , thus indicating a high confidence level of the biological processes identified.

\subsection{Functional Analysis of Top RRE-Enriched and Deficient Molecular Pathways}

In order to functionally characterize top RRE-enriched and deficient molecular pathways, we manually classified them into groups according to their biological functions.

\subsubsection{RRE-Enriched Pathways}

For the top $5 \%$ (155 pathways) we totally identified 14 functional groups (Supplementary Table S7). Thirty-three pathways were classified as general signaling pathways, 22 were connected with cell cycle and mitosis, seven were connected with cell death, 16 were connected with morphogenesis, 12 were connected with lipid metabolism, 13 were connected with cell adhesion, migration and cell-cell interactions, 11 were connected with the immune system, seven were connected with DNA metabolism and chromatin structure, seven were connected with cytoskeleton formation, five were connected with endocytosis, three were connected with RNA synthesis and degradation, two were connected with the metabolism of amino acids and three were connected with sensory perception and neurotransmission. Finally, the group "Other pathways" included 14 stand-alone pathways. 
Table 1. Analysis of human non-coding RNA RE-linked regulation.

\begin{tabular}{|c|c|c|c|c|c|c|}
\hline Group & $\begin{array}{l}\text { Non-Coding } \\
\text { RNA Class }\end{array}$ & $\begin{array}{c}\text { Number of } \\
\text { Non-Coding RNA } \\
\text { Genes in the Sample }\end{array}$ & $\begin{array}{c}\text { Expected Number of } \\
\text { Non-Coding RNA } \\
\text { Genes in the Sample, } \\
\text { Random } \\
\text { Distribution Model }\end{array}$ & $\begin{array}{l}\text { Hypergeometric } p \text {-Value } \\
\text { for Hypothesis That } \\
\text { Non-Coding RNA Genes } \\
\text { are Overrepresented in } \\
\text { the Respective Gene Set }\end{array}$ & $\begin{array}{l}\text { Hypergeometric } p \text {-Value } \\
\text { for Hypothesis That } \\
\text { Non-Coding RNA Genes } \\
\text { are Underrepresented in } \\
\text { the Respective Gene Set }\end{array}$ & Conclusion \\
\hline RRE-enriched & lncRNA & 145 & 74 & $2.22 \times 10^{-8}$ & 0.99999998 & $\begin{array}{c}\text { lncRNAs are } \\
\text { overrepresented }\end{array}$ \\
\hline RRE-deficient & $\operatorname{lncRNA}$ & 54 & 74 & 0.9951 & 0.0049 & $\begin{array}{c}\operatorname{lncRNAs} \text { are } \\
\text { underrepresented }\end{array}$ \\
\hline RRE-enriched & microRNA & 145 & 90 & $3.49 \times 10^{-8}$ & 0.99999997 & $\begin{array}{l}\text { microRNAs are } \\
\text { overrepresented }\end{array}$ \\
\hline RRE-deficient & microRNA & 94 & 90 & 0.33 & 0.67 & $\begin{array}{l}\text { microRNAs are neither } \\
\text { overrepresented nor } \\
\text { underrepresented }\end{array}$ \\
\hline
\end{tabular}


A

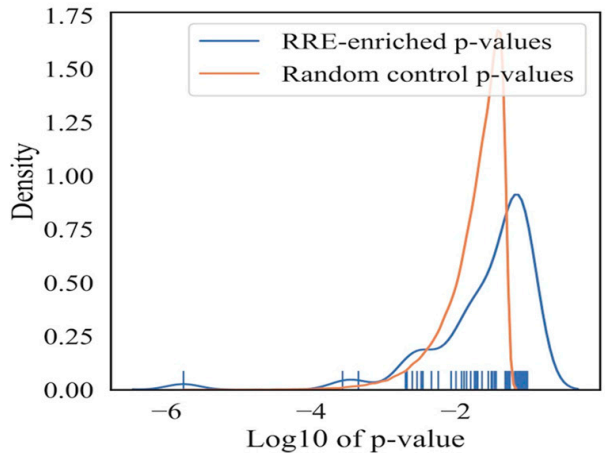

B

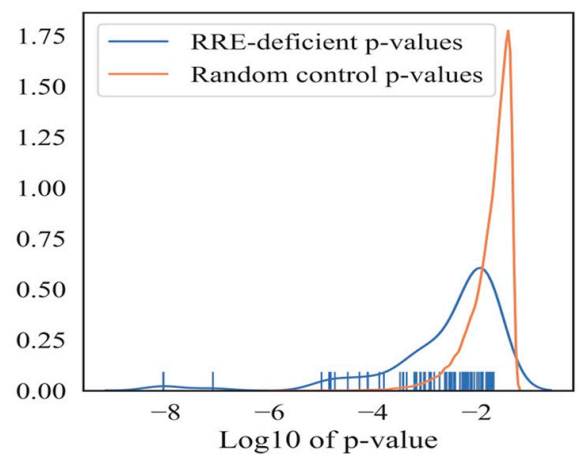

Figure 5. Random control of GO terms enrichment in RRE-enriched (A) and RRE-deficient (B) gene sets.

\subsubsection{RRE-Deficient Pathways}

For the $5 \%$ bottom human pathways we identified 17 functional groups (Supplementary Table S7). Thirty-eight pathways were referred as general signaling pathways, 22 pathways were connected with the immune system, 13 were connected with apoptotic processes, three were connected with the cell cycle, 15 were connected with cell adhesion, migration and intercellular interactions, five were connected with protein aggregation and import, three were connected with translation, protein export and folding, eight were connected with lipids metabolism, eight were connected with the detoxication of xenobiotics, eight were connected with morphogenesis, six were connected with DNA metabolism and chromatin structure, four were connected with the metabolism of amino acids, three were connected with hormone-mediated signaling, three were connected with the metabolism and transport of metal ions, four were connected with endocytosis, and two were connected with formation of the cytoskeleton. Ten pathways were included in the group "Other pathways".

\subsection{Comparison of Gene- and Pathway-Based RRE Data}

We then compared groups of functional processes found to be RRE-enriched or deficient using two types of analyses: based on GO and on molecular pathways (Table 2).

Among the groups that were annotated during both pathway and GO analysis, there were three RRE-enriched groups, seven RRE-deficient groups and five groups with ambiguous trends (four were RRE-enriched according to pathway analysis and RRE-deficient according to GO annotation; contrarily, one group was RRE-enriched by GO annotation and deficient by pathway analysis). Overall, Matthews correlation coefficient for these two analyses was 0.342 , thus indicating their moderate convergence. The consensus groups of molecular processes contained the following pathways and regulatory networks.

\subsubsection{RRE-Enriched Processes}

(1) The "Posttranscriptional silencing by small RNAs" group was identified using Gorilla functional annotation of RRE-enriched genes and included microRNA-mediated gene silencing.

(2) The "DNA Metabolism and Chromatin Structure" group included double strand break DNA repair, transcription-coupled DNA repair, DNA strand displacement and chromatin remodeling processes.

(3) The "Sensory Perception and Neurotransmission" group consisted of olfactory receptors activity (more specifically, class C3 metabotropic glutamate pheromonic receptors activity) and retinoid cycle in cones, which is responsible for color vision in primates.

(4) The "Lipids Metabolism" group contained fatty acid biosynthesis, beta-oxidation and desaturation of fatty acids as well as phospholipase A2 activity and modification of sterols via cytochrome P450. 
Table 2. RRE enrichment of the molecular processes according to GO and pathway analyses.

\begin{tabular}{|c|c|c|c|c|c|}
\hline \multirow{2}{*}{ Group of Processes } & \multicolumn{2}{|c|}{ Pathway Analysis } & \multicolumn{2}{|c|}{ GO Analysis } & \multirow{2}{*}{ Overall Status } \\
\hline & Enriched & Deficient & Enriched & Deficient & \\
\hline Posttranscriptional silencing by small RNAs & 1 & 0 & 1 & 0 & enriched \\
\hline DNA Metabolism and Chromatin Structure & 7 & 6 & 4 & 3 & enriched \\
\hline Sensory Perception and Neurotransmission & 3 & 0 & 2 & 0 & enriched \\
\hline Lipids Metabolism & 12 & 8 & 3 & 0 & enriched \\
\hline Endocytosis & 5 & 4 & 0 & 0 & enriched \\
\hline Immune System & 11 & 21 & 2 & 8 & deficient \\
\hline Protein Ubiquitination and Degradation & 0 & 5 & 0 & 2 & deficient \\
\hline Cell Adhesion, Migration and Interaction & 13 & 15 & 0 & 12 & deficient \\
\hline Metals Metabolism and Ion Transport & 0 & 3 & 3 & 4 & deficient \\
\hline Cell Death & 7 & 13 & 0 & 11 & deficient \\
\hline General Signaling Pathways & 33 & 38 & 0 & 15 & deficient \\
\hline Hormones Signaling Pathways & 0 & 3 & 0 & 4 & deficient \\
\hline Stress Response & 0 & 0 & 0 & 2 & deficient \\
\hline Response to Viruses & 0 & 0 & 0 & 2 & deficient \\
\hline Amino Acids Metabolism & 2 & 4 & 0 & 0 & deficient \\
\hline Detoxication of Xenobiotics & 0 & 8 & 0 & 0 & deficient \\
\hline Protein Aggregation and Import & 0 & 0 & 0 & 7 & deficient \\
\hline Morphogenesis & 16 & 9 & 0 & 45 & ambiguous \\
\hline Cytoskeleton & 7 & 2 & 0 & 7 & ambiguous \\
\hline RNA Synthesis and Degradation & 3 & 0 & 6 & 23 & ambiguous \\
\hline Translation, Protein Export and Folding & 0 & 3 & 3 & 0 & ambiguous \\
\hline Cell Cycle and Mitosis & 22 & 3 & 2 & 6 & ambiguous \\
\hline Other Processes & 14 & 10 & 7 & 18 & ambiguous \\
\hline
\end{tabular}




\subsubsection{RRE-Deficient Processes}

(1) "Immune System" was a heterogenous group of molecular processes such as dendritic cell chemotaxis and cytokine production, IL-1, IL-3, IL-4, TLR and PD-1 signaling, asthma-related signaling and activation of RAS GTPase in B-cells.

(2) The "Protein Ubiquitination and Degradation" group included ubiquitin-ligase activity, K63 polyubiquitin binding (non-degradative signal), protein degradation in proteasomes and autodegradation of E3 ubiquitin-ligase.

(3) The "Cell Adhesion, Migration and Interaction" group included tight junction formation, E-cadherin binding, cell interaction with extracellular matrix (hyaluronanglucosaminidase activity, laminin binding and inhibition of matrix metalloproteinases), MMIF-mediated angiogenesis and platelet aggregation processes.

(4) The "Metals Metabolism and Ion Transport" group consisted of zinc and calcium ion binding, chloride and potassium channels activity.

(5) The "Cell Death" group contained various signaling pathways responsible for activation of apoptosis (p53, MEF2D-mediated apoptosis in T cells and BAD translocation to mitochondria), PTEN-mediated cell cycle arrest leading to apoptosis, caspase cascade and permeabilization of mitochondrial outer membrane.

(6) The "General Signaling Pathways" group included wide variety of signaling pathways such as NK-кB, VEGF, EGF, IGF and mTOR signaling.

(7) The "Hormone Signaling Pathways" group included steroid hormone mediated signaling pathways mediating response to estrogen and testosterone and PELP1 modulation of estrogen receptor activity.

\section{Discussion}

In this study we analyzed the whole genome enrichment profiles of histone mark H3K4me1 in order to identify the extent of RE-linked enhancer regulation of human genes and molecular pathways. The data for five human cell lines of different tissue origin (MCF-7, K562, HepG2, HeLaS3, GM12878) were extracted from the ENCODE repository and analyzed according to the RetroSpect methodology [21,22]. We could only work with the cell lines instead of normal tissues due to public availability of high throughput epigenomic data. The cell lines selected represented four different tissues of human body. However, we found that the histone tags highly correlated between the different cell lines, thus suggesting only minor impact of a tissue-specific component on the data.

Enhancers are long distance-acting cis-regulatory elements that play a central role in mediation of cell type- and cell state- specific variation in gene expression patterns [13]. There are more than $1,000,000$ enhancers in the human genome [14]. The chromatin landscape of enhancers is complex and includes a variety of epigenetic signatures such as histone modifications [53], TFBS [54], DNaseI hypersensitivity sites [55] and short non-coding eRNA expression [56].

Molecular functions of enhancers are primarily dependent on TFs that cooperatively bind to multiple clustered TFBS. This includes lineage specific TFs and sequence-dependent effectors of signaling pathways, which allows for the integration of intrinsic and external signals in gene expression regulation [57]. Previously, we utilized RetroSpect to investigate RE-linked TFBS impact on human gene expression and found a group of conservative molecular processes that were enriched or deficient in RE-linked TFBS regulation [21].

Nevertheless, TFBS is not an enhancer-specific epigenetic feature [57]. Though H3K4me1 is a mark of both active and dormant enhancers [58,59], it is a broad enhancer-specific "window of opportunities" that defines active enhancers as well as those primed to activation and deactivated ones [13]. All other enhancer chromatin markers, such as H3K27ac, appear in the context of pre-existing H3K4me1 [13,59] and disappear after downregulation of enhancer activity [58]. We therefore used H3K4me1 as the 
enhancer hallmark to identify genes and molecular pathways whose regulation is enriched or deficient in RE-linked enhancers-which reflects their regulatory evolution.

Here, we found very high number of non-coding RNAs (microRNA and lncRNA) among the genes enriched in RE-linked enhancer regulation. Moreover, "gene silencing by microRNA and RNA degradation" was the most significant RRE-enriched GO term according to Gorilla software annotation. The same pattern was already observed in the previous study of human genes enriched in RE-linked TFBS regulation [21]. This finding confirms the concept of non-coding RNAs as primary mediators of RE-driven regulatory evolution. Indeed, the non-coding RNAs and REs are tightly interconnected "planets" in the human genome "universe" since RE insertions sometimes generate new non-coding RNA genes that have an evolutionary chance of gaining biological function [60]. Moreover, the non-coding RNA is a source of evolutionary regulatory innovations that dates back to the origin of major Metazoan clades, such as Piwi-interacting RNAs that appeared during the emergence of metazoan multicellularity [61]. Additionally, the evolution of epigenetic repression mediated by small non-coding RNAs (RNA interference) and some other nucleic acids based immune mechanisms were driven by REs and retroviruses as an example of host-pathogen evolutionary arms-race [62]. Therefore, our finding of high RE regulatory load on human non-coding RNAs (detected both at the levels of TFBS and H3K4me1) highlights the vision of non-coding RNAs as the major way of RE-driven regulatory evolution and innovation.

Using RetroSpect functional annotation procedure, we extracted consensus groups of molecular processes that are enriched or deficient in RE-linked enhancer regulation. The set of RRE-enriched processes (three groups) was relatively short compared to the figure of eight groups previously identified by TFBS $\neg$ RetroSpect analysis [21]. This lower number of groups can be explained by the lower number of independent data profiles from ENCODE experimental studies that were included in RetroSpect protocol (one profile for each cell line here and 4260 profiles for different cell lines in [21]). Moreover, the different number of groups of processes can be due to a biological difference of the functional marks investigated: REs can impact gene regulation not only via long-distance acting enhancers, but also via TFBS acting proximal to transcription start site [9]. Therefore, RE-linked TFBS regulatory impact could be broader than the enhancer-only impact.

However, the pattern of molecular processes impacted by RE-linked enhancers agrees well with the previous findings. For example, the high RE-linked enhancer enrichment of the "Sensory perception and neurotransmission" group is in accord with the previously reported enrichment of this group by RE-linked TFBS [21]. This conservative high RRE pattern underlines the fast regulatory evolution of sensory perception and nervous system in the mammalian clade. Indeed, most parts of the mammalian forebrain are linked with the sensory system and have been evolving quickly [63]. Lipids metabolism is another enriched group that also showed quick regulatory evolution in RE-linked TFBS study [21]. The accelerated evolution of human lipidome was shown in several independent lipidomic assays, which was connected with accelerated brain evolution in primate lineage, since lipid composition is crucial for proper functioning of CNS [64]. Moreover, positive selection and coevolution of different lipid metabolism enzymes in primates was detected previously [65] in the context of adaptation of energetic balance and endothermic metabolism [66]. The third RRE-enriched group, DNA metabolism and chromatin structure, primarily contained various processes of DNA repair, which is in agreement with the results of the previous TFBS RetroSpect analysis [21]. This can be explained by redundancy and catalytic promiscuity of DNA repair systems [67].

On the other hand, the conservative (RRE-deficient) molecular processes identified here are also in some aspects congruent with those identified previously by TFBS analysis [21] and with some general evolutionary trends established by other methods. For example, the "Protein ubiquitination and degradation" group is one of the most conservative core intracellular processes [68]. Similarly, the "General signaling pathways" group was found as a group with relatively low regulatory evolution, also in the RetroSpect TFBS analysis [21]. The conservative state of this group is most likely due to an enormous complexity and interdependence of core intracellular signaling in mammals. Deregulation 
of these signaling networks can lead to severe proliferative and developmental disorders in complex long-living organisms such as primates [69]. There are however other means of signaling pathway regulatory evolution such as point mutations and duplications/deletions/translocations of pre-existing regulatory elements [70] that can lead to signaling networks complexity growth [71].

The "Immune system" group was identified here as mostly conservative in contrast to contradictory pattern (RRE-enriched cellular immune response versus RRE-deficient cellular mechanisms of antiviral response) detected previously for the RE-linked TFBS [21]. This difference can be a consequence of lower resolution of RetroSpect methodology for H3K4me1 enhancer mark compared to the larger TFBS datasets (moreTFBS data profiles investigated). Interestingly, in this study, we found three conservative groups important for multicellular organisms, "Cell Adhesion, Migration and Interaction", "Cell Death" and "Hormone Signaling Pathways" are groups that were not found in the TFBS analysis [21]. In the previous RE-linked TFBS study, the impact of these groups was neither RRE-enriched nor deficient. The last group, "Hormone Signaling Pathways", was previously identified as RRE-enriched only in the pathway analysis [21]. We speculate that RE-linked regulation for these groups of processes can be rather promoter-proximal than long distance-acting. Though further correct statistical validation of this hypothesis is required, the underlying biological reason for this asymmetry can be the fact that enhancers are long distance acting elements that require additional cis-acting sequences (insulators and anchor elements) and trans-acting factors (coactivators, CTCF protein, etc.) for the correct looping and activation of the cognate promoter [72,73]. Therefore, emergence of a new RE-linked enhancer could introduce more noise and perturbation into authentic gene regulatory networks than the emergence of a RE-linked TFBS near a pre-existing promoter. Since the above three groups of processes need to be tightly regulated in order to orchestrate development of multicellular organism, the "noisiest" RE insertions could be selectively eliminated or silenced [74]. Intriguingly, the same pattern was seen for the group "Metals Metabolism and Ion Transport" as well.

Despite the marked convergence between current results and TFBS-based analysis [21], as much as 10 groups of molecular processes that were established in the TFBS analysis have not been observed here, and three new groups ("Endocytosis", "Morphogenesis" and "Cytoskeleton") were observed only in the current study. Among groups annotated in both studies, six groups have the same status based on consensus molecular pathways and GO annotation in both studies, five groups have the same status based on single annotation type (at least in one study) and eight groups have different statuses in these two studies. Overall statistics for the two studies are shown in Table 3.

Again, the possible reason for such differences is a lower resolution of RetroSpect for H3K4me1 mark compared to the TFBS datasets. Another possibility is the prevalence of RE-linked promoter-proximal regulation for groups identified in the TFBS study only [21], whereas RE-linked regulation of genes connected with "Endocytosis", "Morphogenesis" and "Cytoskeleton" groups could be long-distance acting rather than promoter-proximal.

Additionally, the possible explanation for the existence of eight groups with different RRE-enrichment statuses is multiple opposite trends in RE-driven regulatory evolution for each of these groups. For example, the "Cell Cycle and Mitosis" group could contain some regulatory networks (such as regulation of cell cycle by certain growth factors) that undergo accelerated regulatory evolution (RRE-enriched), whereas other processes of cell division (such as mitotic spindle formation) could be conservative. Though this scenario is speculative, eukaryotic cell cycle machinery contains both conservative and quickly evolving parts [75]. Further studies are needed to clarify the molecular evolution of processes in groups such as "Immunity", "Metals Metabolism and Ion Transport", "Hormones Signaling Pathways", "Amino Acids Metabolism", "Detoxication of Xenobiotics", "RNA Synthesis and Degradation", "Translation", "Protein Export and Folding", "Cell Cycle" and "Mitosis". 
Table 3. Comparison of isolated groups of processes in current study and in TFBS study [21].

\begin{tabular}{|c|c|c|c|c|c|}
\hline \multirow{3}{*}{ Group of Processes } & \multicolumn{2}{|c|}{ Current Study } & \multicolumn{2}{|r|}{ TFBS Study } & \multirow{3}{*}{ Comment } \\
\hline & \multirow{2}{*}{ Overall Status } & \multirow{2}{*}{$\begin{array}{c}\text { Type of Analysis } \\
\text { (Pathways/GO/Consensus) }\end{array}$} & \multirow{2}{*}{ Status } & \multirow{2}{*}{$\frac{\text { Type of Analysis }}{\text { (Pathways/GO/Consensus) }}$} & \\
\hline & & & & & \\
\hline \multicolumn{6}{|c|}{ Consensus Match } \\
\hline Sensory Perception and Neurotransmission & enriched & consensus & enriched & consensus & \\
\hline Lipids Metabolism & enriched & consensus & enriched & consensus & \\
\hline Protein Ubiquitination and Degradation & deficient & consensus & deficient & consensus & $\begin{array}{l}\text { Corresponds to "Translation and Protein } \\
\text { Quality Control" in the TFBS study }\end{array}$ \\
\hline Posttranscriptional silencing by small RNAs & enriched & consensus & enriched & consensus & $\begin{array}{l}\text { Identified by Gorilla software and } \\
\text { validated using hypergeometric } \\
\text { enrichment in both studies }\end{array}$ \\
\hline DNA Metabolism and Chromatin Structure & enriched & consensus & enriched & consensus & $\begin{array}{c}\text { Corresponds to "DNA repair" in the } \\
\text { TFBS study }\end{array}$ \\
\hline General Signaling Pathways & deficient & consensus & deficient & consensus & \\
\hline \multicolumn{6}{|c|}{ Match by Overall Status } \\
\hline Stress Response & deficient & $\mathrm{GO}$ & deficient & GO & \\
\hline Cell Adhesion, Migration and Interaction & deficient & consensus & deficient & GO & \\
\hline Cell Death & deficient & consensus & deficient & GO & \\
\hline Protein Aggregation and Import & deficient & GO & deficient & GO & $\begin{array}{l}\text { Corresponds to "Protein Localization } \\
\text { and Modification" in the TFBS study }\end{array}$ \\
\hline Response to Viruses & deficient & GO & deficient & consensus & \\
\hline \multicolumn{6}{|c|}{ Does not Match } \\
\hline Immune System & deficient & consensus & ambiguous & - & \\
\hline Metals Metabolism and Ion Transport & deficient & consensus & enriched & GO & \\
\hline Hormones Signaling Pathways & deficient & consensus & enriched & pathways & $\begin{array}{c}\text { Corresponds to "Hormones" in the } \\
\text { TFBS study }\end{array}$ \\
\hline Amino Acids Metabolism & deficient & pathways & enriched & consensus & \\
\hline
\end{tabular}


Table 3. Cont

\begin{tabular}{|c|c|c|c|c|c|}
\hline \multirow{3}{*}{ Group of Processes } & \multicolumn{2}{|c|}{ Current Study } & \multicolumn{2}{|r|}{ TFBS Study } & \multirow{3}{*}{ Comment } \\
\hline & \multirow{2}{*}{ Overall Status } & \multirow{2}{*}{$\frac{\text { Type of Analysis }}{\text { (Pathways/GO/Consensus) }}$} & \multirow{2}{*}{ Status } & \multirow{2}{*}{$\frac{\text { Type of Analysis }}{\text { (Pathways/GO/Consensus) }}$} & \\
\hline & & & & & \\
\hline \multicolumn{6}{|c|}{ Does not Match } \\
\hline Detoxication of Xenobiotics & deficient & pathways & enriched & consensus & \\
\hline RNA Synthesis and Degradation & ambiguous & - & deficient & $\mathrm{GO}$ & \\
\hline Translation, Protein Export and Folding & ambiguous & - & deficient & consensus & $\begin{array}{l}\text { Corresponds to "Translation and Protein } \\
\text { Quality Control" in the TFBS study }\end{array}$ \\
\hline Cell Cycle and Mitosis & ambiguous & - & deficient & GO & \\
\hline \multicolumn{6}{|c|}{ Group Appears Only in one of the Studies } \\
\hline Endocytosis & enriched & pathways & - & & \\
\hline Morphogenesis & ambiguous & - & - & - & \\
\hline Cytoskeleton & ambiguous & - & - & - & \\
\hline $\begin{array}{l}\text { Cellular immune response ( } \mathrm{T} \text { cells and NK } \\
\text { cells) }\end{array}$ & - & - & enriched & consensus & \\
\hline Fertilization & - & - & enriched & consensus & \\
\hline Vitamin metabolism & - & - & enriched & pathways & \\
\hline Molecular transport & - & - & enriched & pathways & \\
\hline $\begin{array}{l}\text { Sulfur metabolism and linked redox } \\
\text { reactions }\end{array}$ & - & - & enriched & pathways & \\
\hline Response to phorbol acetate & - & - & deficient & GO & \\
\hline Electron transfer reactions & - & - & deficient & $\mathrm{GO}$ & \\
\hline Mitochondria & - & - & deficient & GO & \\
\hline $\begin{array}{l}\text { Nucleic Base, Nucleosides and Nucleotides } \\
\text { metabolism }\end{array}$ & - & - & deficient & consensus & \\
\hline Carbohydrates metabolism & - & - & ambiguous & - & \\
\hline
\end{tabular}


In this study, we worked with the human cell line data instead of data for the normal tissues due to public availability of high-throughput profiles for target histone marks and RNA sequencing data for the former. The five cell lines selected for our analysis represented different tissues of human body. However, we found that the histone tags highly correlated between the different cell lines, thus suggesting only minor impact of a tissue-specific component on the data. Availability of novel epigenetic datasets corresponding to normal human tissues would be extremely desirable for further re-analysis of data using RetroSpect pipeline.

Here, we used RetroSpect methodology to analyze a histone mark H3K4me1 in the context of RE-linked transcriptional regulation and RE-linked regulatory evolution. This evidences that RetroSpect is applicable to the different types of functional genome landmarks. We hope that examination of new functional genomic markers using RetroSpect methodology will help building an integrated model of human genome regulatory evolution. We also suggest that the new directions for RetroSpect applications could be non-human organisms such as the model species mouse, zebrafish and drosophila.

\section{Conclusions}

In this study we investigated the regulatory influence of RE-linked enhancer elements on human molecular processes using the previously developed RetroSpect analytic pipeline. We found that the most quickly evolving molecular processes under the regulatory impact of RE-linked enhancers were connected with posttranscriptional silencing by small RNAs, DNA metabolism and chromatin structure, sensory perception/neurotransmission and lipids metabolism. The most conservative processes were dealing with immunity, protein ubiquitination and degradation, cell adhesion, migration and interaction, metals metabolism/ion transport, cell death, general and hormones signaling pathways. There was a significant enrichment of non-coding RNA groups among the genes enriched in RE-linked enhancer regulation. Our findings open an avenue towards building an integral picture of RE-driven regulatory evolution and understanding the origin of regulatory complexity and innovation in humans.

Supplementary Materials: The following are available online at http://www.mdpi.com/2073-4409/8/10/1219/s1. Supplementary Table S1-the list of raw ENCODE data files for each cell line. Supplementary Dataset 2-detailed description of RetroSpect calculation of RE-linked enhancers regulatory impact on human genes and molecular pathways. Supplementary File 3-overall data analysis pipeline used in this study. Supplementary Table S4-RE-linked H3K4me1 histone modifications absolute and normalized enrichment scores GRE and NGRE, respectively. Supplementary Table S5-RRE-enriched and RRE-deficient genes. Supplementary Table S6-molecular pathway RE-linked H3K4me1 histone modifications absolute and normalized enrichment scores PII and NPII, respectively. Supplementary Table S7-manually classified RRE-enriched and RRE-deficient molecular pathways. Supplementary Table S8-manually classified RRE-enriched and RRE-deficient GO terms. Supplementary File 9-results of Gorilla software functional annotation of the RRE-enriched gene set using "Gene Ontology Biological Process" database. Supplementary File 10-results of Gorilla software functional annotation of the RRE-deficient gene set using "Gene Ontology Biological Process" database.

Author Contributions: conceptualization, P.K., D.N. and A.B.; methodology, N.K, D.N. and A.B.; software, N.K., D.N., A.M., A.Z., V.T. and M.S.; validation, N.K., D.N., A.M. and K.P.; formal analysis, N.K., D.N., A.M., K.P., V.T. and M.S.; investigation, D.N.; resources, A.B.; data curation, D.N., K.P., A.Z., V.T. and M.S.; writing-original draft preparation, N.K. and D.N.; writing-review and editing, P.K., A.B., D.N., A.M., K.P. and A.Z.; visualization, N.K., D.N.; supervision, A.B.; project administration, A.B., D.N.; funding acquisition, A.B.

Funding: This study was supported by the Russian Foundation for Basic Research Grant 19-29-01108 and by individual sponsors.

Acknowledgments: We acknowledge the Amazon and Microsoft Azure grants for cloud-based computations, which helped us to complete this study. We thank the Oncobox/OmicsWay research program in machine learning and digital oncology for providing access to software and pathway databases. We thank Professors Philippe Kopylov and Victor Fomin (I.M. Sechenov First Moscow State Medical University), Alexander Markov (Lomonosov Moscow State University) and Michael Galperin (National Center for Biotechnology Information (NCBI)) for their help with the organization of the First Hackathon on Evolutionary Bioinformatics that served as the platform for organizing and performing this study.

Conflicts of Interest: The authors declare no conflict of interests. 


\section{References}

1. Rodić, N.; Burns, K.H. Long interspersed element-1 (LINE-1): Passenger or driver in human neoplasms? PLoS Genet. 2013, 9, e1003402. [CrossRef]

2. Lander, E.S.; Linton, L.M.; Birren, B.; Nusbaum, C.; Zody, M.C.; Baldwin, J.; Devon, K.; Dewar, K.; Doyle, M.; FitzHugh, W.; et al. Initial sequencing and analysis of the human genome. Nature 2001, 409, 860-921.

3. Policarpi, C.; Crepaldi, L.; Brookes, E.; Nitarska, J.; French, S.M.; Coatti, A.; Riccio, A. Enhancer SINEs Link Pol III to Pol II Transcription in Neurons. Cell Rep. 2017, 21, 2879-2894. [CrossRef]

4. Suntsova, M.; Gogvadze, E.V.; Salozhin, S.; Gaifullin, N.; Eroshkin, F.; Dmitriev, S.E.; Martynova, N.; Kulikov, K.; Malakhova, G.; Tukhbatova, G.; et al. Human-specific endogenous retroviral insert serves as an enhancer for the schizophrenia-linked gene PRODH. Proc. Natl. Acad. Sci. USA 2013, 110, 19472-19477. [CrossRef]

5. Pan, G.; Ameur, A.; Enroth, S.; Bysani, M.; Nord, H.; Cavalli, M.; Essand, M.; Gyllensten, U.; Wadelius, C. PATZ1 down-regulates FADS1 by binding to rs174557 and is opposed by SP1/SREBP1c. Nucleic Acids Res. 2017, 45, 2408-2422. [CrossRef]

6. Suntsova, M.; Garazha, A.; Ivanova, A.; Kaminsky, D.; Zhavoronkov, A.; Buzdin, A. Molecular functions of human endogenous retroviruses in health and disease. Cell. Mol. Life Sci. 2015, 72, 3653-3675. [CrossRef]

7. Chuong, E.B.; Elde, N.C.; Feschotte, C. Regulatory evolution of innate immunity through co-option of endogenous retroviruses. Science 2016, 351, 1083-1087. [CrossRef]

8. Dunn-fletcher, C.E.; Muglia, L.M.; Pavlicev, M.; Wolf, G.; Sun, M.A.; Hu, Y.C.; Huffman, E.; Tumukuntala, S.; Thiele, K.; Mukherjee, A.; et al. Anthropoid primate-specific retroviral element THE1B controls expression of CRH in placenta and alters gestation length. PLoS Biol. 2018, 16, e2006337. [CrossRef] [PubMed]

9. Gogvadze, E.; Buzdin, A. Retroelements and their impact on genome evolution and functioning. Cell. Mol. Life Sci. 2009, 66, 3727-3742. [CrossRef] [PubMed]

10. Mita, P.; Boeke, J.D. How retrotransposons shape genome regulation. Curr. Opin. Genet. Dev. 2016, 37, 90-100. [CrossRef] [PubMed]

11. Marnetto, D.; Mantica, F.; Molineris, I.; Grassi, E.; Pesando, I.; Provero, P. Evolutionary Rewiring of Human Regulatory Networks by Waves of Genome Expansion. Am. J. Hum. Genet. 2018, 102, 207-218. [CrossRef] [PubMed]

12. Blinka, S.; Reimer, M.H.; Pulakanti, K.; Pinello, L.; Yuan, G.C.; Rao, S. Identification of Transcribed Enhancers by Genome-Wide Chromatin Immunoprecipitation Sequencing. Methods Mol. Biol. 2017, 1468, 91-109. [PubMed]

13. Calo, E.; Wysocka, J. Modification of enhancer chromatin: What, how, and why? Mol. Cell. 2013, 49, 825-837. [CrossRef] [PubMed]

14. ENCODE Project Consortium. An integrated encyclopedia of DNA elements in the human genome. Nature 2012, 489, 57-74. [CrossRef] [PubMed]

15. Siggens, L.; Ekwall, K. Epigenetics, chromatin and genome organization: Recent advances from the ENCODE project. J. Intern. Med. 2014, 276, 201-214. [CrossRef]

16. Long, H.K.; Prescott, S.L.; Wysocka, J. Ever-Changing Landscapes: Transcriptional Enhancers in Development and Evolution. Cell 2016, 167, 1170-1187. [CrossRef]

17. Rubinstein, M.; De souza, F.S. Evolution of transcriptional enhancers and animal diversity. Philos. Trans. R. Soc. Lond. B Biol. Sci. 2013, 368, 20130017. [CrossRef]

18. Carelli, F.N.; Liechti, A.; Halbert, J.; Warnefors, M.; Kaessmann, H. Repurposing of promoters and enhancers during mammalian evolution. Nat. Commun. 2018, 9, 4066. [CrossRef]

19. Emera, D.; Yin, J.; Reilly, S.K.; Gockley, J.; Noonan, J.P. Origin and evolution of developmental enhancers in the mammalian neocortex. Proc. Natl. Acad. Sci. USA 2016, 113, E2617-E2626. [CrossRef]

20. Nikitin, D.; Penzar, D.; Garazha, A.; Sorokin, M.; Tkachev, V.; Borisov, N.; Poltorak, A.; Prassolov, V.; Buzdin, A.A. Profiling of Human Molecular Pathways Affected by Retrotransposons at the Level of Regulation by Transcription Factor Proteins. Front. Immunol. 2018, 9, 30. [CrossRef]

21. Nikitin, D.; Garazha, A.; Sorokin, M.; Penzar, D.; Tkachev, V.; Markov, A.; Gaifullin, N.; Borger, P.; Poltorak, A.; Buzdin, A. Retroelement-Linked Transcription Factor Binding Patterns Point to Quickly Developing Molecular Pathways in Human Evolution. Cells 2019, 8, 130. [CrossRef] [PubMed] 
22. Pontarotti, P. Evolution, Origin of Life, Concepts and Methods, 1st ed.; Springer International Publishing: New York, NY, USA, 2019.

23. Danino, Y.M.; Even, D.; Ideses, D.; Juven-gershon, T. The core promoter: At the heart of gene expression. Biochim. Biophys. Acta 2015, 1849, 1116-1131. [CrossRef] [PubMed]

24. Zhang, Z.D.; Paccanaro, A.; Fu, Y.; Weissman, S.; Weng, Z.; Chang, J.; Snyder, M.; Gerstein, M.B. Statistical analysis of the genomic distribution and correlation of regulatory elements in the ENCODE regions. Genome Res. 2007, 17, 787-797. [CrossRef] [PubMed]

25. Buzdin, A.; Sorokin, M.; Garazha, A.; Sekacheva, M.; Kim, E.; Zhukov, N.; Wang, Y.; Li, X.; Kar, S.; Hartmann, C.; et al. Molecular pathway activation-New type of biomarkers for tumor morphology and personalized selection of target drugs. Semin Cancer Biol. 2018, 53, 110-124. [CrossRef] [PubMed]

26. Mooney, M.A.; Wilmot, B. Gene set analysis: A step-by-step guide. Am. J. Med. Genet. B. Neuropsychiatr. Genet. 2015, 168, 517-527. [CrossRef] [PubMed]

27. Aliper, A.M.; Korzinkin, M.B.; Kuzmina, N.B.; Zenin, A.A.; Venkova, L.S.; Smirnov, P.Y.; Zhavoronkov, A.A.; Buzdin, A.A.; Borisov, N.M. Mathematical Justification of Expression-Based Pathway Activation Scoring (PAS). Methods Mol. Biol. 2017, 1613, 31-51. [PubMed]

28. Zolotovskaia, M.A.; Sorokin, M.I.; Roumiantsev, S.A.; Borisov, N.M.; Buzdin, A.A. Pathway Instability Is an Effective New Mutation-Based Type of Cancer Biomarkers. Front. Oncol. 2019, 8, 658. [CrossRef]

29. Kanehisa, M.; Goto, S. KEGG: Kyoto encyclopedia of genes and genomes. Nucleic Acids Res. 2000, $28,27-30$. [CrossRef]

30. Fabregat, A.; Sidiropoulos, K.; Garapati, P.; Sidiropoulos, K.; Gillespie, M.; Garapati, P.; Haw, R.; Jassal, B.; Korninger, F.; May, B.; et al. The Reactome pathway Knowledgebase. Nucleic Acids Res. 2016, 44, D481-D487. [CrossRef]

31. Gaudet, P.; Dessimoz, C. Gene Ontology: Pitfalls, Biases, and Remedies. Methods Mol. Biol. 2017, 1446, 189-205.

32. Miletić, A.; Krmpotić, A.; Jonjić, S. The evolutionary arms race between NK cells and viruses: Who gets the short end of the stick? Eur. J. Immunol. 2013, 43, 867-877. [CrossRef] [PubMed]

33. Kawamura, S. Color vision diversity and significance in primates inferred from genetic and field studies. Genes Genomics 2016, 38, 779-791. [CrossRef] [PubMed]

34. ENCODE Database. Available online: https://www.encodeproject.org/ (accessed on 16 August 2019).

35. ENCODE Database. Histone ChIP-seq protocol. Available online: https://www.encodeproject.org/chip-seq/ histone/ (accessed on 16 August 2019).

36. ENCODE Database, ChIP-seq Read Mapping. Available online: https://www.encodeproject.org/pipelines/ ENCPL220NBH/ (accessed on 16 August 2019).

37. ENCODE Database, ChIP-seq Peak Calling. Available online: https://www.encodeproject.org/pipelines/ ENCPL272XAE/ (accessed on 16 August 2019).

38. RepeatMasker. Available online: http://www.repeatmasker.org (accessed on 16 August 2019).

39. UCSC Browser, Human Genome. Available online: https://genome.ucsc.edu/cgi-bin/hgs (accessed on 16 August 2019).

40. BioCarta. Available online: https://cgap.nci.nih.gov/Pathways/BioCarta_Pathways (accessed on 16 August 2019).

41. KEGG. Available online: http://www.genome.jp/kegg/ (accessed on 16 August 2019).

42. National Cancer Institute. Available online: https://cactus.nci.nih.gov/ncicadd/about.htm (accessed on 16 August 2019).

43. Reactome. Available online: http://reactome.org (accessed on 16 August 2019).

44. Pathway Central. Available online: http://www.sabiosciences.com/pathwaycentral.php (accessed on 16 August 2019).

45. Buzdin, A.; Sorokin, M.; Poddubskaya, E.; Borisov, N. High-Throughput Mutation Data Now Complement Transcriptomic Profiling: Advances in Molecular Pathway Activation Analysis Approach in Cancer Biology. Cancer Inform. 2019, 18, 838-844. [CrossRef] [PubMed]

46. DAVID Functional Annotation Bioinformatics Microarray Analysis. Available online: https://david.ncifcrf. gov/ (accessed on 16 August 2019).

47. USCS Genome Browser. Available online: https://genome.ucsc.edu/ (accessed on 16 August 2019). 
48. Huang, W.; Sherman, B.T.; Lempicki, R.A. Bioinformatics enrichment tools: Paths toward the comprehensive functional analysis of large gene lists. Nucleic Acids Res. 2009, 37, 1-13. [CrossRef]

49. Python Seaborn package. Available online: http://seaborn.pydata.org/ (accessed on 16 August 2019).

50. Python Sklearn package. Available online: https://scikit-learn.org/stable/documentation.html (accessed on 16 August 2019).

51. Gene Ontology documentation. Available online: http://geneontology.org/docs/ontology-documentation/ (accessed on 16 August 2019).

52. GOrilla-A tool for Identifying Enriched GO Terms. Available online: http://cbl-gorilla.cs.technion.ac.il (accessed on 16 August 2019).

53. Heintzman, N.D.; Hon, G.C.; Hawkins, R.D.; Kheradpour, P.; Stark, A.; Harp, L.F.; Ye, Z.; Lee, L.K.; Stuart, R.K.; Ching, C.W.; et al. Histone modifications at human enhancers reflect global cell-type-specific gene expression. Nature 2009, 459, 108-112. [CrossRef] [PubMed]

54. Spitz, F.; Furlong, E.E. Transcription factors: From enhancer binding to developmental control. Nat Rev Genet. 2012, 13, 613-626. [CrossRef]

55. Gross, D.S.; Garrard, W.T. Nuclease hypersensitive sites in chromatin. Annu Rev Biochem. 1988, 57, $159-197$. [CrossRef]

56. Natoli, G.; Andrau, J.C. Noncoding transcription at enhancers: General principles and functional models. Annu. Rev. Genet. 2012, 46, 1-19. [CrossRef]

57. Inukai, S.; Kock, K.H.; Bulyk, M.L. Transcription factor-DNA binding: Beyond binding site motifs. Curr. Opin. Genet. Dev. 2017, 43, 110-119. [CrossRef]

58. Bogdanovic, O.; Fernandez-miñán, A.; Tena, J.J.; de la Calle-Mustienes, E.; Hidalgo, C.; van Kruysbergen, I.; van Heeringen, S.J.; Veenstra, G.J.; Gómez-Skarmeta, J.L. Dynamics of enhancer chromatin signatures mark the transition from pluripotency to cell specification during embryogenesis. Genome Res. 2012, 22, 2043-2053. [CrossRef]

59. Bonn, S.; Zinzen, R.P.; Girardot, C.; Gustafson, E.H.; Perez-Gonzalez, A.; Delhomme, N.; Ghavi-Helm, Y.; Wilczyński, B.; Riddell, A.; Furlong, E.E. Tissue-specific analysis of chromatin state identifies temporal signatures of enhancer activity during embryonic development. Nat. Genet. 2012, 44, 148-156. [CrossRef]

60. Ganesh, S.; Svoboda, P. Retrotransposon-associated long non-coding RNAs in mice and men. Pflugers Arch. 2016, 468, 1049-1060. [CrossRef]

61. Gaiti, F.; Calcino, A.D.; Tanurdžić, M.; Degnan, B.M. Origin and evolution of the metazoan non-coding regulatory genome. Dev. Biol. 2017, 427, 193-202. [CrossRef]

62. Hartmann, G. Nucleic Acid Immunity. Adv. Immunol. 2017, 133, 121-169.

63. Kaas, J.H. The evolution of complex sensory systems in mammals. J. Exp. Biol. 1989, 146, 165-176.

64. Khrameeva, E.; Kurochkin, I.; Bozek, K.; Giavalisco, P.; Khaitovich, P. Lipidome Evolution in Mammalian Tissues. Mol. Biol. Evol. 2018, 35, 1947-1957. [CrossRef]

65. Lin, B.; Yuan, L.; Chen, J. Selection pressure drives the co-evolution of several lipid metabolism genes in mammals. Chin. Sci. Bull. 2012, 57, 877. [CrossRef]

66. Hulbert, A.J.; Else, P.L. Evolution of mammalian endothermic metabolism: Mitochondrial activity and cell composition. Am. J. Physiol. 1989, 256, R63-R69. [CrossRef]

67. O'brien, P.J. Catalytic promiscuity and the divergent evolution of DNA repair enzymes. Chem. Rev. 2006, 106, 720-752. [CrossRef]

68. Allan, D.C.; Phillips, J.C. Why Ubiquitin Has Not Evolved. Int. J. Mol. Sci. 2017, 18, 1995. [CrossRef]

69. Van dam, T.J.; Zwartkruis, F.J.; Bos, J.L.; Snel, B. Evolution of the TOR pathway. J. Mol. Evol. 2011, 73, $209-220$. [CrossRef]

70. Signor, S.A.; Nuzhdin, S.V. The Evolution of Gene Expression in cis and trans. Trends Genet. 2018, 34, 532-544. [CrossRef]

71. Soyer, O.S.; Bonhoeffer, S. Evolution of complexity in signaling pathways. Proc. Natl. Acad. Sci. USA 2006, 103, 16337-16342. [CrossRef]

72. Ko, J.Y.; Oh, S.; Yoo, K.H. Functional Enhancers as Master Regulators of Tissue-Specific Gene Regulation and Cancer Development. Mol. Cells. 2017, 40, 169-177. 
73. Zabidi, M.A.; Stark, A. Regulatory Enhancer-Core-Promoter Communication via Transcription Factors and Cofactors. Trends Genet. 2016, 32, 801-814. [CrossRef]

74. Hancks, D.C.; Kazazian, H.H. Active human retrotransposons: Variation and disease. Curr. Opin. Genet. Dev. 2012, 22, 191-203. [CrossRef]

75. Harashima, H.; Dissmeyer, N.; Schnittger, A. Cell cycle control across the eukaryotic kingdom. Trends Cell Biol. 2013, 23, 345-356. [CrossRef]

(C) 2019 by the authors. Licensee MDPI, Basel, Switzerland. This article is an open access article distributed under the terms and conditions of the Creative Commons Attribution (CC BY) license (http://creativecommons.org/licenses/by/4.0/). 